\title{
GABAergic Signaling within a Limbic-Hypothalamic Circuit Integrates Social and Anxiety-Like Behavior with Stress Reactivity
}

\author{
Brent Myers*,', Eduardo Carvalho-Netto', Dayna Wick-Carlson', Christine Wu', Sam Naser', \\ Matia B Solomon', Yvonne M Ulrich-Lai' and James P Herman' \\ 'Department of Psychiatry and Behavioral Neuroscience, University of Cincinnati, Cincinnati, OH, USA
}

\begin{abstract}
The posterior hypothalamic nucleus $(\mathrm{PH})$ stimulates autonomic stress responses. However, the role of the $\mathrm{PH}$ in behavioral correlates of psychiatric illness, such as social and anxiety-like behavior, is largely unexplored, as is the neurochemistry of PH connectivity with limbic and neuroendocrine systems. Thus, the current study tested the hypothesis that GABAergic signaling within the PH is a critical link between forebrain behavior-regulatory nuclei and the neuroendocrine hypothalamus, integrating social and anxiety-related behaviors with physiological stress reactivity. To address this hypothesis, GABA receptor pharmacology was used to locally inhibit or disinhibit the $\mathrm{PH}$ immediately before behavioral measures of social and anxiety-like behavior in rats. Limbic connectivity of the PH was then established by simultaneous co-injection of anterograde and retrograde tracers. Further, the role of PH GABAergic signaling in neuroendocrine stress responses was tested via inhibition/disinhibition of the $\mathrm{PH}$. These studies determined a prominent role for the $\mathrm{PH}$ in the expression of anxiety-related behaviors and social withdrawal. Histological analyses revealed divergent stress-activated limbic input to the $\mathrm{PH}$, emanating predominantly from the prefrontal cortex, lateral septum, and amygdala. $\mathrm{PH}$ projections also targeted both parvicellular and magnocellular peptidergic neurons in the paraventricular and supraoptic hypothalamus. Further, GABA $A$ receptor pharmacology determined an excitatory effect of the $\mathrm{PH}$ on neuroendocrine responses to stress. These data indicate that the $\mathrm{PH}$ represents an important stressintegrative center, regulating behavioral processes and connecting the limbic forebrain with neuroendocrine systems. Moreover, the PH appears to be uniquely situated to have a role in stress-related pathologies associated with limbic-hypothalamic dysfunction.

Neuropsychopharmacology (2016) 4I, I530-1539; doi:I0.1038/npp.2015.3II; published online 9 December 2015
\end{abstract}

\section{INTRODUCTION}

Appropriate appraisal of stress, defined as a real or perceived threat to homeostasis or well-being, is critical for organismal survival. Primary physiological responses to stress mobilize energy and provide resources to promote adaptation (Herman, 2013; Myers et al, 2014). However, aberrant activation of stress systems increases susceptibility to multiple pathological conditions, including neuropsychiatric disorders (Sudheimer et al, 2015). Importantly, brain sites responsible for the appraisal of stressful information, such as the medial prefrontal cortex (mPFC), hippocampus, and amygdala, do not provide substantial direct innervation of stress-effector sites (Herman et al, 2003; Ulrich-Lai and Herman, 2009). Thus, brain nuclei providing direct innervation of neuroendocrine and pre-autonomic neurons are hypothesized to integrate and relay limbic stress information

* Correspondence: Dr B Myers, Department of Psychiatry and Behavioral Neuroscience, University of Cincinnati, 2170 East Galbraith Road, Cincinnati, OH 45237, USA, Tel: + I 5135583029 , Fax: + I 513558 9104, E-mail: brent.myers@uc.edu

Received 13 June 2015; revised 21 August 2015; accepted 29 September 2015; accepted article preview online 7 October 2015
(Myers et al, 2012; Ulrich-Lai and Herman, 2009). Based on this hypothesis, nuclei that provide direct excitatory input to endocrine and autonomic cell groups would be positioned to assimilate limbic information, generate stress reactivity, and, ultimately, guide affective behaviors.

The posterior nucleus of the hypothalamus $(\mathrm{PH})$ is positioned at a pivotal point of intersection between descending limbic circuits and behavioral/physiological effector systems. The $\mathrm{PH}$ provides glutamatergic innervation of the paraventricular hypothalamus (PVN) (Ulrich-Lai et al, 2011) and stimulates sympathetic responses including thermogenesis, increased heart rate, and elevated blood pressure (Abrahamson and Moore, 2001; DiMicco et al, 1986; Lisa et al, 1989). Moreover, the infralimbic (IL) mPFC, a critical regulator of stress responsivity and mood (Hamani et al, 2010; McKlveen et al, 2013), provides dense innervation of the PH (Vertes, 2004) and is activated in parallel with the $\mathrm{PH}$ following chronic variable stress (Flak et al, 2012). Collectively, these data suggest that interactions between the $\mathrm{PH}$ and limbic forebrain could represent a neurocircuit critical for stress adaptation and, perhaps, stress pathology.

The current study tests the hypothesis that the $\mathrm{PH}$ is an important integrative node that translates forebrain inputs 
into behavioral and neuroendocrine reactivity. Specifically, these studies query the function of GABAergic signaling in the $\mathrm{PH}$ as it relates to social and anxiety-related behaviors. Further, we simultaneously investigate stress-activated forebrain inputs to the $\mathrm{PH}$ as well as the peptidergic phenotype of neuroendocrine cells targeted by the $\mathrm{PH}$ using bidirectional pathway mapping. Here we identify input to specific endocrine cell groups to elucidate mechanisms by which the $\mathrm{PH}$ may regulate physiological reactivity. Based on this connectivity, we also explore the role of the $\mathrm{PH}$ in neuroendocrine stress responses, with the ultimate goal of determining the neural substrates integrating affective behavior with stress reactivity.

\section{MATERIALS AND METHODS}

\section{Animals}

Male Sprague-Dawley rats (250-300 g; Harlan, Indianapolis, IN) were housed individually in standard rat cages and acclimated for 1 week before initiation of experiments. Rats were maintained in a temperature- and humidity-controlled room (12:12 light:dark, lights on $0600 \mathrm{~h}$, off at $1800 \mathrm{~h}$ ) with food and water available ad libitum. All experimental procedures and protocols were approved by the University of Cincinnati Institutional Animal Care and Use Committee.

\section{Experiment 1: Social and Anxiety-Related Behavior}

Rats $(N=36)$ received bilateral cannula implantation in the $\mathrm{PH}$ (Supplementary Information). Briefly, guide cannulae (26 gauge; Plastics One, Roanoke, VA) were stereotaxically implanted at $4.0 \mathrm{~mm}$ posterior to bregma, $0.4 \mathrm{~mm}$ left and right of midline, and $5.0 \mathrm{~mm}$ ventral from the dura. Injectors were cut to extend $2.0 \mathrm{~mm}$ ventral from the tip of the guide cannula. These coordinates were chosen to target the dorsal portion of the $\mathrm{PH}$, substantially dorsal and caudal to the dorsal medial nucleus of the hypothalamus. Animals were then divided into three groups ( $n=12$ each): saline controls, $\mathrm{GABA}_{\mathrm{A}}$ agonist muscimol (100 ng in saline; Sigma), or $\mathrm{GABA}_{\mathrm{A}}$ antagonist bicuculline methiodide (BMI; $10 \mathrm{ng}$ in saline; Sigma). Doses of GABA agonists and antagonists were based on previously published reports (DiMicco et al, 1986; Lisa et al, 1989). Injections were performed under light manual restraint immediately preceding the social interaction test. Following injections, rats were placed into a clean, novel, standard-sized cage with a weight-matched male Sprague-Dawley rat for $10 \mathrm{~min}$ (Krause et al, 2011). Behavior was quantified based on the frequency and duration of social behavior (either neutral, aggressive, or submissive) and nonsocial behavior (Supplementary Information). Group means were calculated for social and nonsocial behavior, while aggressive and submissive behaviors were calculated as percent of time engaged with the interactor. These were taken as indices of sociality, aggression, and avoidance. Animals were allowed 3 days to recover before being counter-balanced, injected, and exposed to the open field test/novel object interaction (Supplementary Information), assaying indices of avoidance, neophobia, and risk (Belzung and Griebel, 2001). Following a 3 day recovery period, animals were injected before testing in the elevated plus maze, a well-characterized assay of anxiety-like behavior (File et al, 2004). Arm entries, time spent in arms, and the number of end explorations (all four feet in the outer half of the open arms) were quantified (Supplementary Information). All injections were ordered so that animals received each treatment only once, a design chosen to conserve animal use. Although animals were permitted no less than 3 days of recovery between injections, we cannot exclude an effect of previous drug exposure on subsequent behavior. Behavioral observations were recorded by a video camera mounted directly above each apparatus. The social interaction test was scored by a blinded observer, while the open field test/novel object interaction and elevated plus maze were scored using Clever TopScan Software (CleverSys, Reston, Virginia). Placement of microinjections was determined based on (Swanson, 2004).

\section{Experiment 2: Circuit Analysis}

Iontophoretic co-injection of the anterograde tracer Phaseolus vulgaris leucoagglutinin (Pha-L) and retrograde tracer cholera toxin beta subunit $(\mathrm{CTb})$ into the $\mathrm{PH}$ (Supplementary Information) was employed to simultaneously determine the forebrain stress-activated input to the $\mathrm{PH}$ and neurochemical phenotype of cells receiving innervation from the $\mathrm{PH}$. Rats received injections followed by a 8-10 day recovery period to allow for tracer transport. Animals then received restraint stress followed by euthanasia, perfusion, and brain collection (Supplementary Information). A total of 27 animals were injected to generate 5 cases suitable for analysis (uptake and transport of both tracers with injections confined to the boundaries of the $\mathrm{PH}$, as determined by Swanson (2004). This dual tract-tracing method has been characterized previously (Coolen and Wood, 1998; Thompson and Swanson, 2010).

Detailed immunohistochemical protocols are described in the Supplementary Information. Single immunolabeling of Pha-L or CTb used either goat anti-Pha-L or goat anti-CTb primary antibody. Dual fluorescent immunolabeling was performed as described (Supplementary Information) for the following reactions: $\mathrm{CTb}$ and $\mathrm{Pha}-\mathrm{L}, \mathrm{CTb}$ and Fos, and Pha- $\mathrm{L}$ with either oxytocin, arginine vasopressin (AVP), or corticotropin-releasing hormone $(\mathrm{CRH})$.

For quantification of $\mathrm{CTb}$, Fos, and $\mathrm{CTb} / \mathrm{Fos}$ colocalization (Supplementary Information), digital images were captured using optical sectioning ( $\times 40$ magnification) to allow for determination of co-localization within a given $z$-plane $(0.5 \mu \mathrm{m}$ thickness). For mapping $\mathrm{PH}$ output (Supplementary Information), $z$-stacks of $0.5 \mu \mathrm{m}$ optical sections were captured at high power ( $\times 63$ magnification). Image stacks were used for analysis of terminal to soma/dendrite appositions. Appositions were further verified by three-dimensional renderings of image stacks containing targeted cells using Volocity software (Improvision).

\section{Experiment 3: Hypothalamic-Pituitary-Adrenocortical (HPA) Axis Stress Responses}

A cohort of 23 rats received bilateral cannulation of the $\mathrm{PH}$ as in Experiment 1. After recovery, animals were divided into two groups ( $n=10-13 /$ group) and received injections of 
saline or the $\mathrm{GABA}_{\mathrm{A}}$ agonist muscimol immediately preceding restraint stress (Supplementary Information). Restraint was used as a psychogenic stressor for analysis of HPA activation, as determined by plasma adrenocorticotropic hormone (ACTH) and corticosterone levels. Stress response assessment was carried out as described (Supplementary Information). Immediately following injection, animals were placed in well-ventilated Plexiglas restraint tubes for $30 \mathrm{~min}$ with tail clip blood samples $(200-300 \mu \mathrm{l})$ collected. Radioimmunoassay was used to determine plasma hormone concentrations (Supplementary Information). Rats recovered for 2 weeks before being counter-balanced and divided into two new groups (n=11-12/group) that received injections of saline or the $\mathrm{GABA}_{\mathrm{A}}$ antagonist $\mathrm{BMI}$ before restraint. Following another 2 week period, animals were divided into two counter-balanced groups $(n=10$ each) receiving saline or BMI in the absence of stress to examine Fos induction after $\mathrm{PH}$ disinhibition both as an indicator of successful treatment and a measure of PVN activation. For Fos immunohistochemistry, tissue was processed as described (Supplementary Information). Digital images were collected for quantification of Fos-positive immunoreactive nuclei in the $\mathrm{PH}$ and $\mathrm{PVN}$ using light microscopy at the $\times 20$ objective. The number of Fos-immunoreactive cells was calculated using Scion Image software (Supplementary Information).

\section{Experiment 4: Prefrontal Inputs to PH GABAergic Neurons}

A group of rats $(n=5)$ received unilateral injections of the anterograde tracer dextran amine (BDA; Invitrogen) (Veenman et al, 1992) into the IL (Supplementary Information). Animals recovered for 12-14 days before processing brains for histology as described for experiment 2 . Following dual florescent immunolabeling of $\mathrm{BDA}$ and GABA, analysis of IL terminal appositions on to GABAlabeled cells in the $\mathrm{PH}$ was carried out using $\times 63 z$-sections (Supplementary Information).

\section{Statistical Analysis}

All data are presented as mean \pm SEM. All behavioral data were analyzed using one-way ANOVA with treatment as a factor. Treatment differences were assessed using Fisher's LSD post test. Time-course data for plasma ACTH and corticosterone were analyzed using two-way repeated measures ANOVA with treatment and time as factors. Post-hoc tests were only conducted when significant effects of treatment or treatment $\times$ time interactions were reported. Treatment differences at individual time points were assessed using Fisher's LSD post test. Adrenal responsivity (concentration of corticosterone divided by the $\log$ of the concentration of ACTH (Ulrich-Lai and Engeland, 2002)), and Fos cell counts were analyzed using $t$-tests. Values that fell outside $2 \times$ the standard deviation from the mean were considered outliers and removed from analysis, as were all cases with injections outside the boundaries of the $\mathrm{PH}$.

\section{RESULTS}

\section{Involvement of $\mathrm{PH}$ in Social and Anxiety-Related Behaviors}

Histological investigation confirmed that all rats included in behavioral analyses received microinjections within the boundaries of the PH (Supplementary Figure S1). Analysis of social interaction indicated an effect of treatment $(\mathrm{F}(2,33)=4.2$, $p<0.05)$, as BMI $(n=12)$ decreased $(p<0.05)$ social behavior (Figure 1a) compared with both saline $(n=12)$ and muscimol $(n=12)$. This corresponded with an increase $(p<0.05)$ in nonsocial behavior following BMI injection (Supplementary Figure S2). Furthermore, there was an effect of treatment on the percentage of aggressive interactions $(\mathrm{F}(2,33)=5.8, p<0.01)$ as $\mathrm{PH}$ disinhibition increased $(p<0.05)$ aggressive interactions compared with saline (Figure 1b). There were no significant differences between muscimol and saline on any of the social behaviors analyzed. These data suggest that tonic GABAergic inhibition within the $\mathrm{PH}$ prevents social avoidance and, perhaps, aggression.

Time spent in the center $v s$ the periphery of the open field was used as a measure of anxiety-like behavior. There was an effect of treatment $(\mathrm{F}(2,32)=8.3, p<0.001)$ on latency to enter the center of the open field, carried by decreased latency in the muscimol group $(n=12)$ relative to saline $(n=11$; Figure 1c). An effect of treatment $(\mathrm{F}(2,32)=5.5, p<0.01)$ was also evident on time spent in the center of the open field. BMI $(n=12)$ decreased center time compared with saline $(p<0.05)$ and muscimol $(p<0.01$; Figure 1d). Importantly, there was no effect of treatment on total distance traveled in the open field (Supplementary Figure S3), indicating that decreased exploratory behavior is not related to effects on locomotion. These findings indicate that $\mathrm{PH}$ GABAergic signaling is necessary and sufficient for inhibition of anxietyrelated behaviors, including avoidance and risk aversion.

Within the center of the open field, a $10 \mathrm{~cm}$ square surrounding a novel object was defined as an interaction zone to query the role of the $\mathrm{PH}$ in behaviors related to novelty. There was an effect of treatment $(\mathrm{F}(2,32)=4.2$, $p<0.05)$ on the latency to enter the interaction zone as well as time spent interacting with the object (nose-poking or sniffing $) \quad(\mathrm{F}(2,32)=4.1, \quad p<0.05)$. Muscimol injections $(n=12)$ decreased $(p<0.05)$ latency to enter the interaction zone compared with saline ( $n=11$; Figure 1e) and increased the time spent contacting the novel object compared with BMI $(n=12)$ and saline $(p<0.05$; Figure 1f). These results indicate a role for the $\mathrm{PH}$ in neophobia, an additional anxiety-like behavior. The combined open field/novel object interaction tests were used to investigate exploratory behavior in a novel environment; however, we cannot exclude the possibility that the novel object influenced open field results. Nonetheless, these data collectively indicate an avoidant behavioral phenotype.

Time spent in the open arms of the elevated plus maze evaluates anxiety-like behavior. Analysis revealed an effect of treatment $(\mathrm{F}(2,32)=14.4, p<0.001)$ on open arm time. $\mathrm{PH}$ muscimol injections $(n=12 ; p<0.05)$ increased time spent in the open arms compared with saline $(n=11$; Figure 1g, Supplementary Figure S4), whereas BMI injections $(n=12 ; p<0.01)$ decreased open arm time compared with saline. Additionally, there was a treatment effect $(F(2,32)=4.4$, 
a

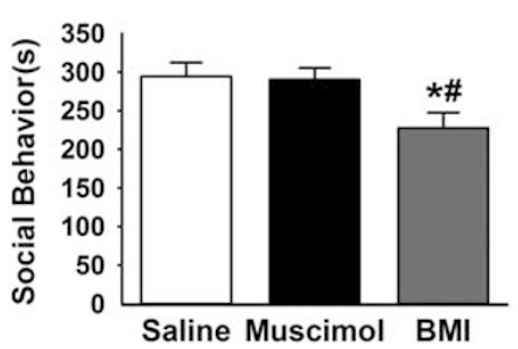

Social Interaction

b

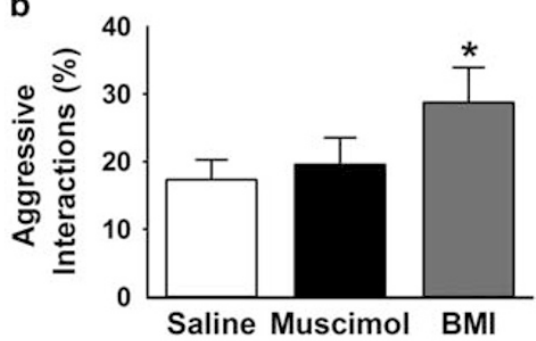

Open Field Test
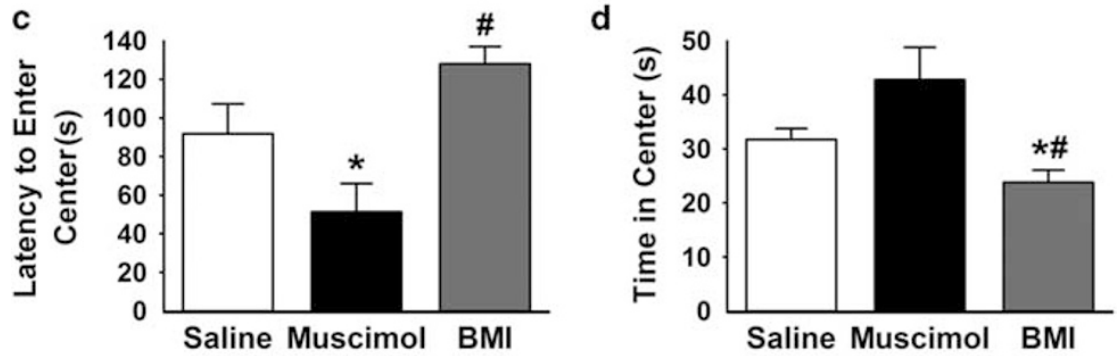

Novel Object Interaction
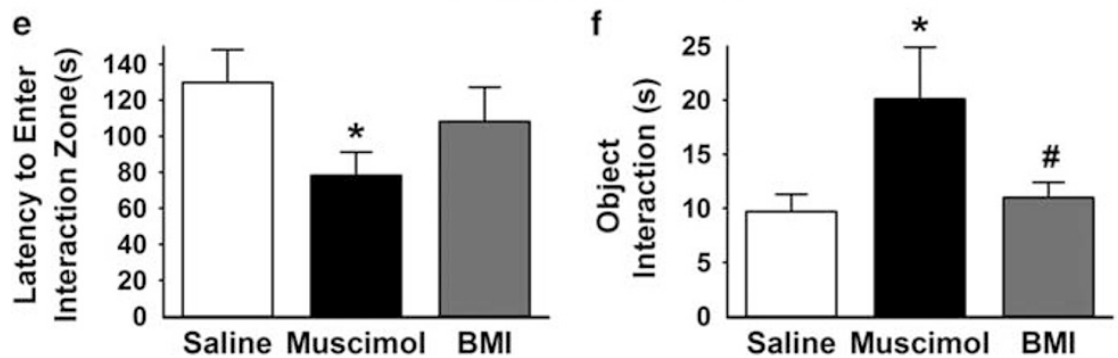

Elevated Plus Maze

g

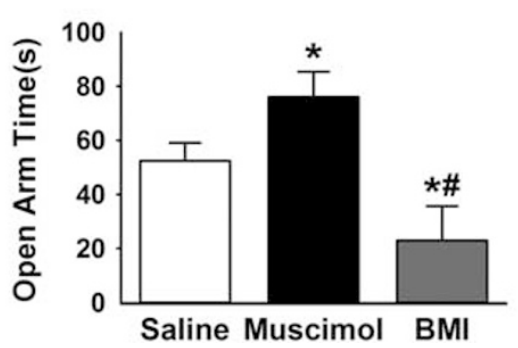

h

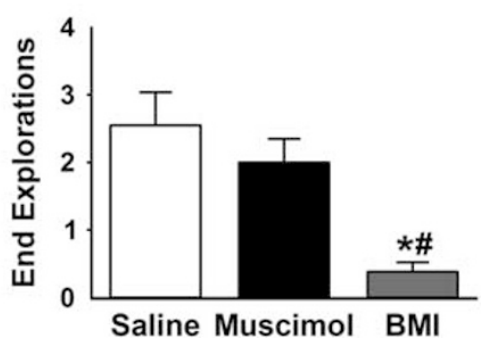

Figure I Involvement of PH in social and anxiety-related behaviors. BMI injections significantly decreased social behavior in the social interaction test (a) and increased the percentage of aggressive interactions (b). In the open field test, muscimol injections in the PH significantly decreased the latency to enter the center of the field compared with saline and BMI (c), while BMI injections significantly decreased the total time spent in the center of the field compared with saline and muscimol (d). Muscimol injections also significantly decreased the latency to enter the $10 \mathrm{~cm}$ square interaction zone surrounding a novel object (e) and significantly increased the amount of time spent interacting (sniffing or nose-poking) with the novel object ( $f$ ). Injections of muscimol into the PH significantly increased time spent in the open arms of the elevated plus maze (g). In contrast, BMl injections significantly decreased time in the open arms, as well as exploration of the ends of open arms (h). $n=1 \mid-12$ /group, ${ }^{*} p<0.05$ compared with saline, ${ }^{\#} p<0.05$ compared with muscimol. BMl, bicuculline methiodide; PH, posterior hypothalamic nucleus.

$p<0.05)$ on the exploration of the ends of the open arms. BMI decreased $(p<0.05)$ the number of open arm end explorations compared with saline and muscimol (Figure 1h). Thus, GABAergic signaling in $\mathrm{PH}$ is necessary and sufficient for controlling anxiety-like behavior in the elevated plus maze.

\section{Identification of Stress-Activated Limbic Input to the PH}

We identified five cases where tracer deposits were localized within the defined boundaries of the PH (Figure 2a; 13). In all five cases the retrograde tracer $\mathrm{CTb}$ was present in the mPFC (Figure 2b), with dense labeling in the IL division (Figure 2c). The presence of $\mathrm{CTb} /$ Fos co-localization was examined throughout the brain to identify stress-activated inputs to the $\mathrm{PH}$. The greatest numbers of $\mathrm{CTb} / \mathrm{Fos}$ co-labeled neurons were in the IL (Figure 2d), lateral septum (LS; Figure 2e), and medial amygdala (MeA; Figure 2f). Quantification of CTb-Fos co-localization (Figure $2 \mathrm{~g}$ and $\mathrm{h}$ ) revealed that the $\mathrm{mPFC}$, LS, and amygdala are primary stress-activated $\mathrm{PH}$ inputs. The MeA provided the most 
abundant projections from the amygdala; however, the central, cortical, and basomedial divisions of the amygdala also generated stress-activated inputs to the $\mathrm{PH}$. Interestingly, other key limbic nuclei, including the basolateral amygdala and hippocampus, exhibited very sparse CTb labeling (not shown). There was extensive input to the $\mathrm{PH}$ from other hypothalamic nuclei and the BST. While some hypothalamic inputs were stress-activated (preoptic area and anterior hypothalamus) there was no co-localization of Fos with the extensive CTb deposits in the BST (Supplementary Table S1). To confirm that projections from the IL, LS, and MeA were not related to off-target retrograde labeling, the anterograde tracer Pha-L was injected in these sites and terminal fibers were observed in the $\mathrm{PH}$ (Figure $2 \mathrm{i}-\mathrm{k}$ ).

\section{Projections from the PH to Peptide-Producing Cells}

Similar to previous reports (Ulrich-Lai et al, 2011), we observed extensive PVN innervation by the PH (Figure $3 \mathrm{a}$ ). Pha-L-labeled fibers and terminals were also present in areas providing input to the $\mathrm{PH}$, including the IL (Figure $3 \mathrm{~b}$ ), LS, and MeA. CTb/Pha-L colabeling in the IL revealed that CTblabeled cells ( $\mathrm{PH}$-projecting) received appositions from Pha$\mathrm{L}$ fibers (Figure $3 \mathrm{c}$ ), indicating reciprocal connectivity. This level of interconnectedness suggests a high level of integration within the circuit.

To determine the neurochemistry of the PVN neurons receiving input from the $\mathrm{PH}$, we first examined Pha-L appositions on CRH-labeled parvicellular neurons (Figure 3d). PVN neurons positive for CRH received dense innervation from the PH. PVN cells expressing AVP and OT also exhibited Pha-L appositions (Figure $3 e$ and f). Highmagnification, single-cell, 3-D renderings of parvicellular AVP (Figure $3 \mathrm{~g}$ ) and magnocellular oxytocin (Figure $3 \mathrm{~h}$ ) neurons were used to confirm putative appositions. Based on the heavy innervation of AVP- and oxytocin-expressing cells in the PVN, the supraoptic nucleus (SON) was also examined for Pha-L appositions. Projections from the $\mathrm{PH}$ densely innervated the SON, targeting AVP (Figure 3i) and oxytocin (Figure 3j) neurons. Combined with previous studies describing the glutamatergic nature of $\mathrm{PH}$ projections to the PVN (Ulrich-Lai et al, 2011), these findings indicate that PH output may regulate a variety of neuroendocrine process, including HPA axis activity and social behavior.

\section{Role of PH in HPA Axis Responses to Stress}

$\mathrm{GABA}_{\mathrm{A}}$-mediated inhibition of the $\mathrm{PH}$ produced a significant treatment $\times$ time interaction $(\mathrm{F}(3,57)=3.3, p<0.05)$ on plasma ACTH (Figure 4a) responses to restraint stress. Post-hoc analysis indicated that muscimol $(n=13)$ inhibited
ACTH release at $15 \mathrm{~min}(p<0.05)$ and $30 \mathrm{~min}(p<0.01)$ after the initiation of stress compared with saline $(n=10)$. However, there was no effect of muscimol treatment on plasma corticosterone levels (Figure 4b). Dissociation of ACTH and corticosterone is not uncommon and is thought to be mediated by sympathetic effects on adrenal responsivity to ACTH (Bornstein et al, 2008). Analysis of peak corticosterone concentration as a function of the log of peak ACTH concentration is a standard, yet indirect, measure of adrenal responsivity (Ulrich-Lai and Engeland, 2002). Here, there was an increase $(t(21)=3.4, p<0.01)$ in adrenal responsivity following the initial inhibition of ACTH release (Figure 4c).

Following $\mathrm{GABA}_{\mathrm{A}}$-mediated disinhibition of $\mathrm{PH}$, there was a treatment $\times$ time interaction $(\mathrm{F}(3,57)=3.1, p<0.05)$ on plasma ACTH (Figure 4d). Compared with saline $(n=11)$, BMI $(n=12)$ increased $(p<0.05) \mathrm{ACTH}$ at $30 \mathrm{~min}$ after the initiation of restraint. BMI also induced a treatment effect $(\mathrm{F}(1,59)=4.3, p<0.05)$ on plasma corticosterone responses to stress. Specifically, BMI $(n=12)$ increased $(p<0.05)$ corticosterone compared with saline $(n=11)$ at $15 \mathrm{~min}$ (Figure $4 \mathrm{e})$. There was no effect of $\mathrm{PH}$ disinhibition on adrenal responsivity (Figure 4f). This suggests that changes in adrenal responsiveness observed with muscimol may relate to sympathetic compensation for low ACTH in response to stress. The experiments utilizing muscimol and BMI were conducted and analyzed sequentially, preventing direct statistical comparison; however, there was an apparent decrease in plasma ACTH in salinetreated animals across experiments. This may reflect habituation in the response to restraint and/or may have resulted from the additional 2 weeks of handling and acclimatization. Furthermore, differential activation of the $\mathrm{PH}$ by habituating $v s$ non-habituating stress paradigms (Flak et al, 2012) suggests the region could have a role in ACTH habituation.

Histological analysis indicated that on-site injections generally targeted the dorsal aspects of the $\mathrm{PH}$ with some rostral-caudal spread (Figure $4 \mathrm{~g}$ ). There was an increase $(t(18)=7.7, p<0.001)$ in the number of Fos-immunoreactive cells in animals treated with BMI compared with saline ( $n=10$ /group; Figure $4 \mathrm{~h})$ in the $\mathrm{PH}$, indicating that BMI effectively activates neurons in this region. BMI injections in the $\mathrm{PH}$ also increased $(t(18)=15.4, p<0.001)$ the number of Fos-immunoreactive cells in the PVN compared with saline ( $n=10$ /group; Figure 4i), providing functional evidence for an excitatory circuit from the $\mathrm{PH}$ to the PVN.

\section{Prefrontal-Targeting of PH GABAergic Neurons}

Based on data above indicating that GABAergic inhibition within the $\mathrm{PH}$ restrains anxiety-like behavior and endocrine stress reactivity, we tested whether inputs from the IL to the $\mathrm{PH}$ target local GABA neurons in the PH. Anterograde tracer

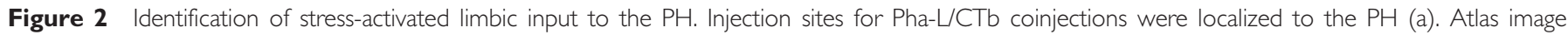

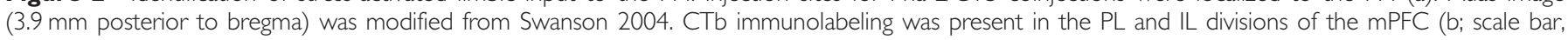

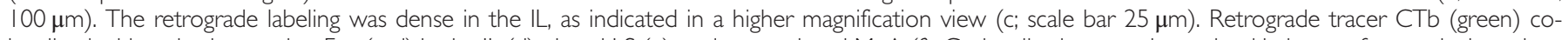

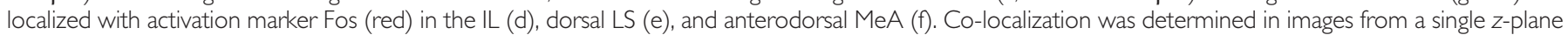

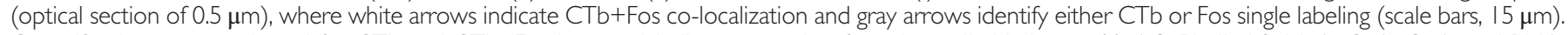

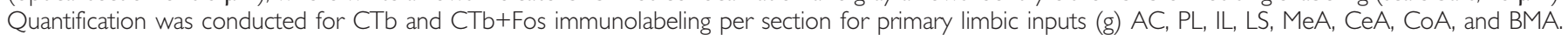

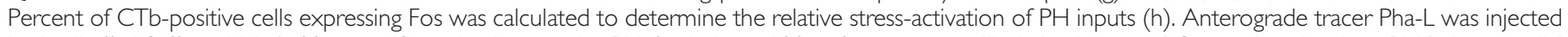

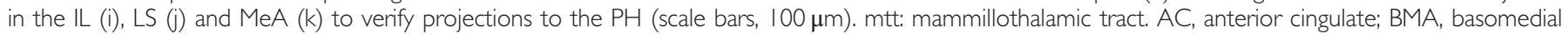

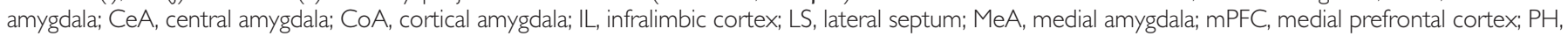
posterior hypothalamic nucleus; Pha-L, Phaseolus vulgaris leucoagglutinin; PL, prelimbic cortex.
} 
(BDA) injected in the IL (Figure 5a) labeled terminals apposing $\mathrm{PH}$ GABA-expressing neurons (Figure 5b). This indicates that glutamatergic output from the IL is anatomically positioned to drive inhibitory neurons within the $\mathrm{PH}$, representing a potential novel mechanism for prefrontal tuning of behavior and stress reactivity.
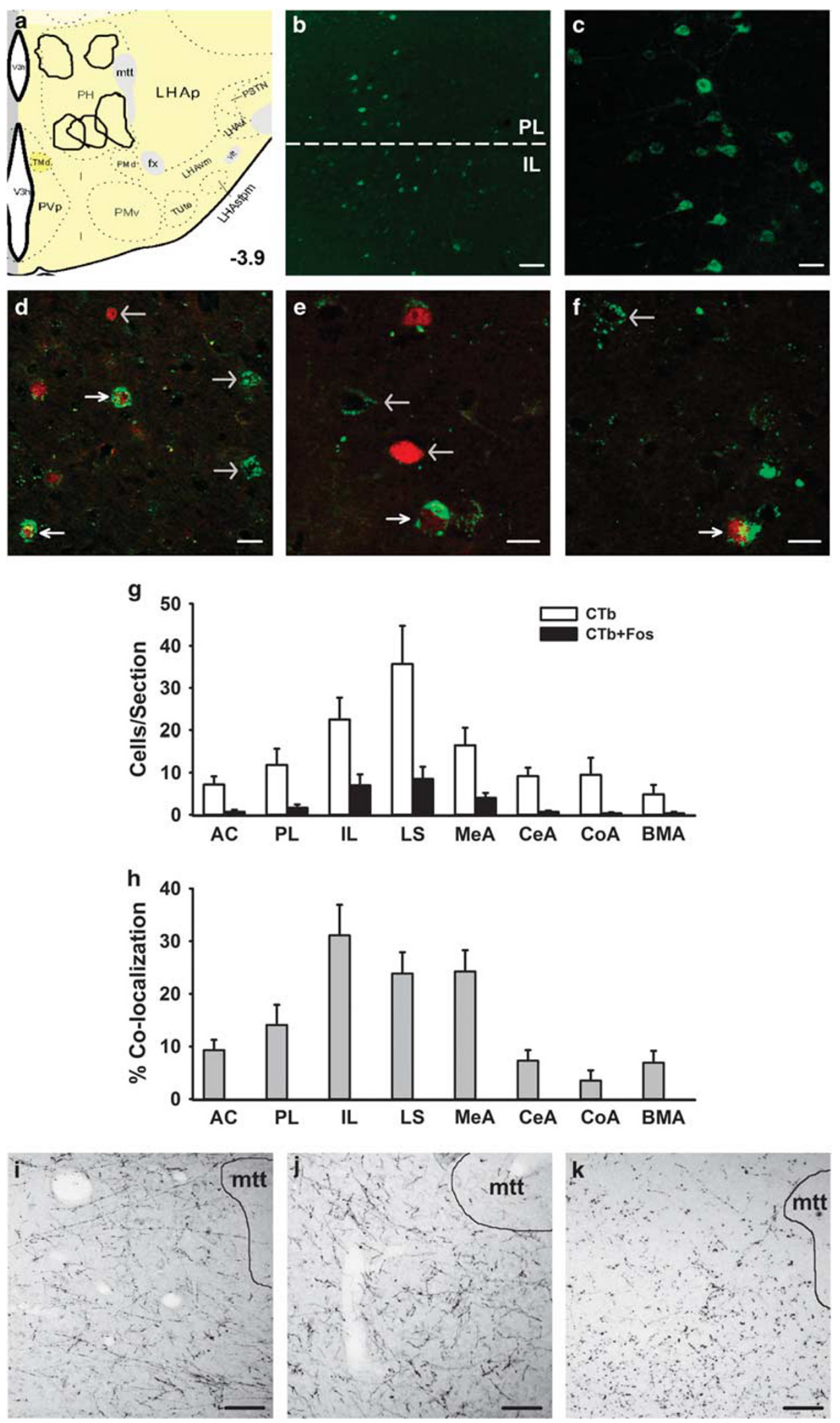


\section{DISCUSSION}

The current study establishes an important role for the $\mathrm{PH}$ in coordination of behavioral and neuroendocrine responses, likely mediated through interactions with key limbic forebrain circuitry. Our studies demonstrate that GABAergic
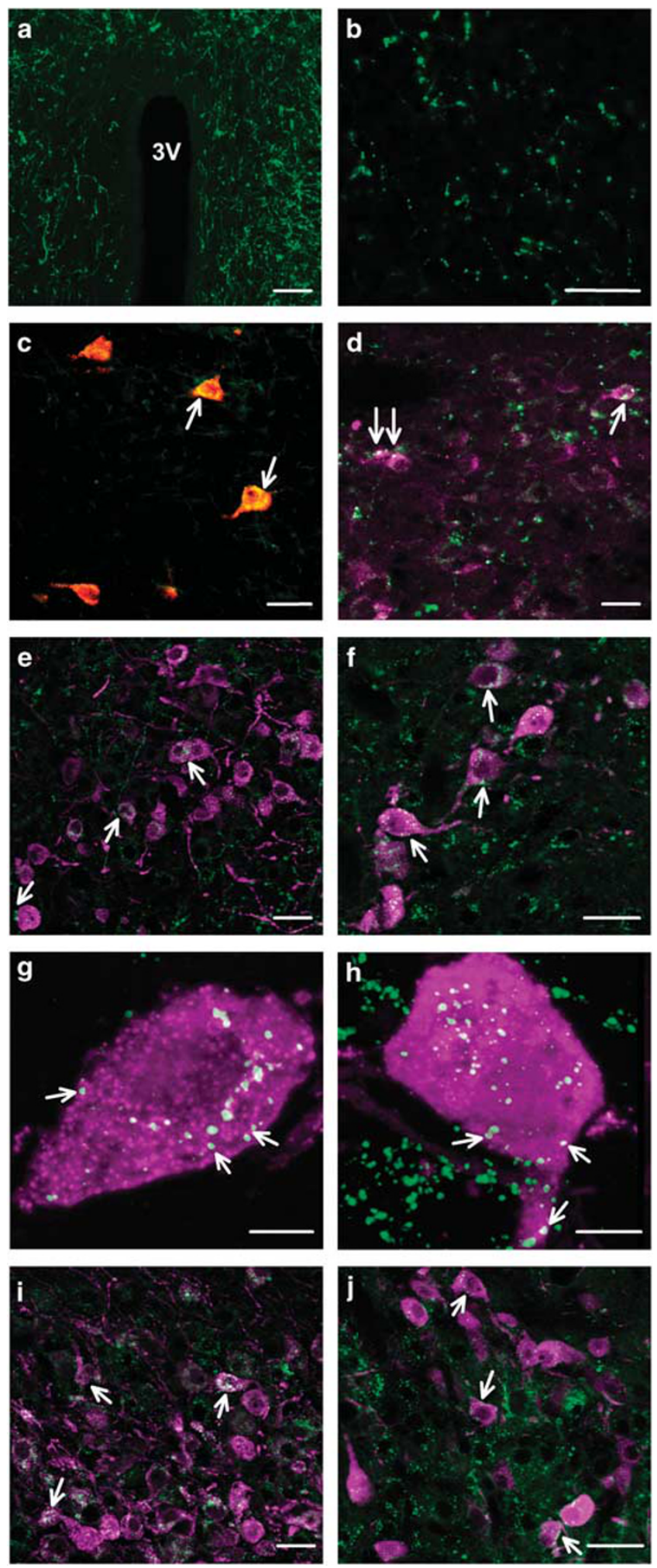

signaling within the $\mathrm{PH}$ is critical for reducing anxiety-like behavior, social withdrawal, and HPA axis stress responses. Given the ability of the $\mathrm{PH}$ to provide glutamatergic drive to behavioral and endocrine effector systems (Ulrich-Lai $e t$ al, 2011), these findings indicate that PH output stimulates an array of avoidance behaviors while simultaneously activating the HPA axis. We also identified excitatory input to $\mathrm{PH}$ GABAergic cells from the IL mPFC, highlighting a potential mechanism whereby cortico-limbic inputs can tune local inhibition within the $\mathrm{PH}$ and perhaps restrain anxiety-like behavior and stress reactivity (Figure $5 \mathrm{c}$ ). Collectively, the data suggest that the $\mathrm{PH}$ functions as a clearinghouse for integrated flight-or-flight responses, and may be an important component of behavioral and physiological responses initiated by upstream limbic circuitry.

Antagonism of $\mathrm{GABA}_{\mathrm{A}}$ in the $\mathrm{PH}$ increased local Fos expression in otherwise unstressed animals. Behavioral analysis following $\mathrm{PH}$ disinhibition indicated that $\mathrm{PH}$ output was sufficient to enhance anxiety-related behaviors characterized by social and risk-avoidance, as well as aggression. Conversely, $\mathrm{GABA}_{\mathrm{A}}$-mediated inhibition of the $\mathrm{PH}$ was anxiolytic, suggesting the $\mathrm{PH}$ is necessary for novelty avoidance and inhibition of behavioral responses. In aggregate, our data suggest that $\mathrm{PH}$ output promotes anxiety-like behaviors in novel contexts and enhances social avoidance. These conclusions are supported by previous findings indicating the sufficiency of the $\mathrm{PH}$ for 'flight' responses in a novel environment and enhanced responding in an active shock avoidance paradigm (Shekhar and DiMicco, 1987; Shekhar et al, 1987). Interestingly, chronic variable stress exposure activates the PH (Flak et al, 2012), suggesting the $\mathrm{PH}$ may represent a stress-recruited node that integrates limbic inputs to alter behavior in the face of prolonged adversity. Our data also suggest that the behavioral influences of the mPFC and amygdala, which include anxiety-like behavior and associative fear (Ebner et al, 2004; Sotres-Bayon and Quirk, 2010), may be mediated, at least in part, by the PH. Future studies focused on interactions of prefrontal and amygdala inputs with local circuit integration in the $\mathrm{PH}$ may yield novel insight into neurobehavioral disorders characterized by avoidance and/or fear. Additionally, the resident-intruder paradigm could provide more details on $\mathrm{PH}$ involvement in aggressionrelated behaviors.

Divergent limbic forebrain sites targeted the $\mathrm{PH}$, including stress-activated input from areas known to be important for stress and behavior such as the MPFC, amygdala, and LS.

Figure 3 Projections from the PH to peptide-producing cells. Pha-Llabeled projections from the PH densely innervated the PVN (a; scale bar, $100 \mu \mathrm{m})$. The PH also innervated the IL (b; scale bar, I00 $\mu \mathrm{m})$. CTb-labeled (red) cells in the IL received input from Pha-L-labeled (green) fibers (c; scale bar, $15 \mu \mathrm{m}$ ), indicating reciprocal regional connectivity. $\mathrm{PH}$ projections (green) to the PVN targeted CRH- (d), AVP- (e), and oxytocin-labeled ( $\mathrm{f}$ ) neurons (magenta; scale bars, I $5 \mu \mathrm{m}$ ). White arrows identify representative putative appositions. Single-cell 3-D reconstructions illustrate Pha-L (green) appositions (white arrows) onto parvicelluar AVP (magenta; g) and magnocellular oxytocin (magenta; h) neurons (scale bars, $5 \mu \mathrm{m}$ ). PH projections (green) also targeted AVP- (i; magenta) and oxytocin-labeled (j; magenta) cells in the SON (scale bars, I5 $\mu \mathrm{m}$ ). AVP, arginine vasopressin; $\mathrm{CRH}$, corticotropin-releasing hormone; CTb, cholera toxin beta subunit; $\mathrm{PH}$, posterior hypothalamic nucleus; Pha-L, Phaseolus vulgaris leucoagglutinin; PVN, paraventricular hypothalamus; SON, supraoptic nucleus. 
a
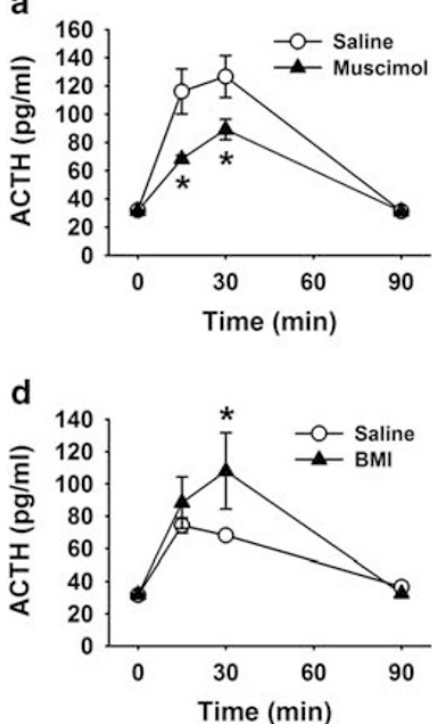

g

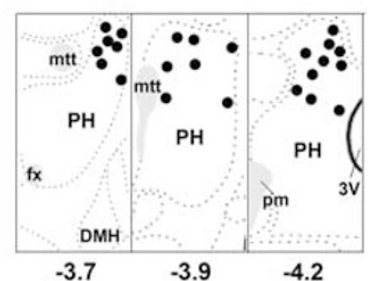

b

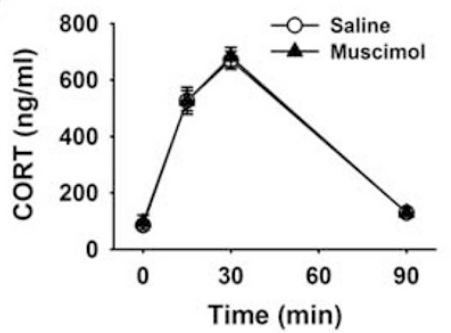

e

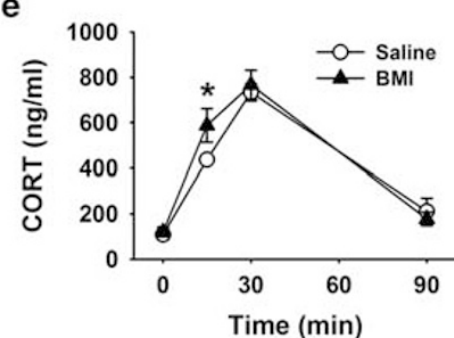

h

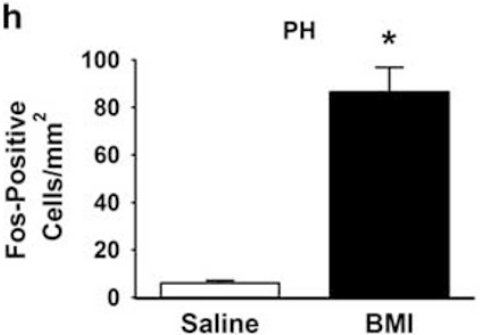

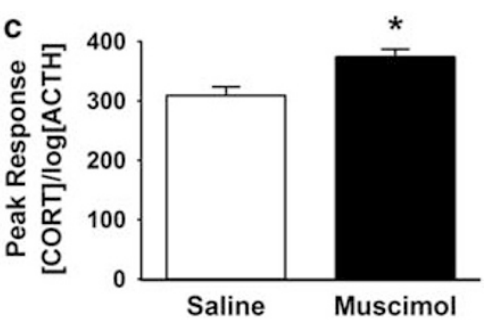

f

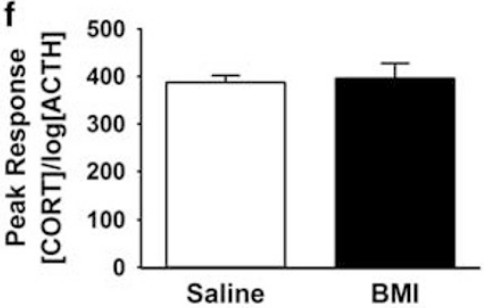

i

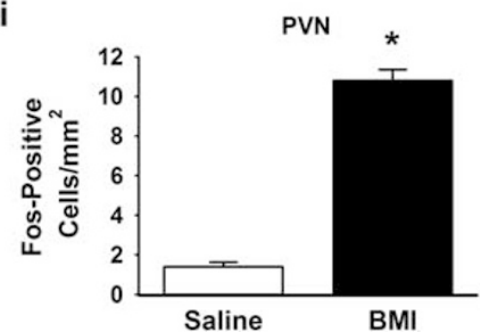

Figure 4 Role of $\mathrm{PH}$ in HPA axis responses to stress. The GABA agonist muscimol injected into the $\mathrm{PH}$ significantly inhibited the $\mathrm{ACTH}$ response to restraint (a). There was no difference in the corticosterone response to stress (b). Analysis of adrenal responsivity revealed that adrenal responsiveness was enhanced during stress (c), possibly to compensate for low ACTH. Injections of the GABA antagonist BMl into the PH significantly increased ACTH (d) and corticosterone (e) responses to stress, without altering adrenal responsivity (f). Microinjection sites were localized to the dorsal aspects of the PH (g) based on the atlas of Swanson (2004). Distances listed are posterior to bregma, $\mathrm{mtt}, \mathrm{fx}, \mathrm{pm}, \mathrm{DMH}$. BMI injections in the PH of unstressed rats significantly increased the number of Fos-positive cells compared with saline (h). Disinhibition of the PH also increased the number of Fos-positive cells in the PVN of unstressed animals (i). $n=10$-13/group, * $p<0.05$ compared with saline. ACTH, adrenocorticotropic hormone; BMI, bicuculline methiodide; DMH: dorsomedial hypothalamus; fx: fornix; HPA, hypothalamic-pituitary-adrenocortical; mtt: mammilothalamic tract; pm: principal mammillary tract; PH, posterior hypothalamic nucleus; PVN, paraventricular hypothalamus.
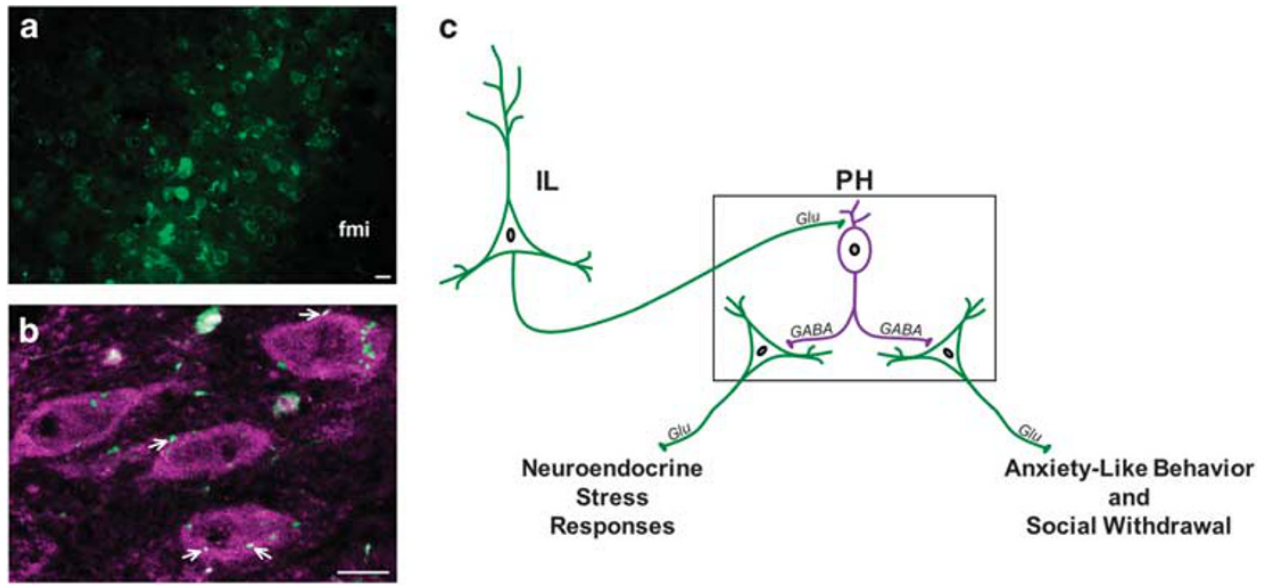

Figure $5 \mathrm{IL}$ projections to GABAergic PH neurons. BDA was injected into the IL (a) and BDA-labeled IL terminals (green) apposed GABA-expressing neurons (magenta) in the PH (b). fmi: forceps minor of the corpus callosum. Co-localization was determined in images from a single z-plane (optical section of $0.5 \mu \mathrm{m}$ ), where white arrows indicate BDA+GABA co-localization (scale bars, $10 \mu \mathrm{m}$ ). Summary of findings and hypothesized circuitry (c). The current study details the activity of local GABA neurons (magenta) in the $\mathrm{PH}$ to constrain excitatory output from the PH (green) that promotes anxiety-like behavior, social withdrawal, and neuroendocrine stress responses. A potential circuit accounting for prefrontal activation of local inhibitory mechanisms within PH is based on infralimbic terminal appositions onto PH GABA neurons. BDA, biotinylated dextran amine; IL, infralimbic cortex; PH, posterior hypothalamic nucleus. 
Recent studies indicate that glucocorticoid signaling in the mPFC, particularly the IL, has an important role in inhibition of HPA axis and behavioral responses to stressors (McKlveen et al, 2013). Our studies indicate that stressactivated IL neurons innervate the $\mathrm{PH}$, implicating the $\mathrm{PH}$ as an intermediary nucleus that may relay the influence of the IL to stress-effector sites. The heavy reciprocal communication between the IL and $\mathrm{PH}$ suggests that these regions form an important integrated circuit, providing both drive and feedback. Furthermore, we found IL terminal appositions onto PH GABA neurons, which could represent a novel mechanism for prefrontal inhibition of behavioral and physiological stress reactivity. However, it is important to note that mRNA expression in the $\mathrm{PH}$ indicates the region is predominately glutamatergic with GABAergic cells representing an intermingled minority population posited to engage in intranuclear regulation (Ziegler et al, 2002). Based on the density of IL innervation of the $\mathrm{PH}$, it is likely that the IL also targets glutamatergic cells. However, due to the lack of a suitable commercially available antibody for immunohistochemically labeling hypothalamic glutamate cell bodies, we were unable to assess IL inputs to this cell population.

Various amygdalar nuclei, including the medial, central, cortical, and basomedial, also innervated the $\mathrm{PH}$. The amygdala has long been considered a nexus of anxiety- and fear-related behaviors (Phelps and LeDoux, 2005). However, functional connectivity between the amygdala and caudal aspects of the hypothalamus has not been widely studied. Although networks within the extended amygdala have been proposed to mediate anxiety and fear behaviors (Davis, 1997), the present findings suggest that amygdala-PH communication may be a component of the circuits responsible for these behaviors. Although less studied, the LS inhibits HPA axis stress responses as well as aggression. Specifically, lesions of the LS enhance HPA axis activity, and produce the well-known 'septal rage' syndrome of hyperaggresion (Schnurr, 1972; Singewald et al, 2011). Given our social behavior data and previous reports of septal GABAergic projections (Myers et al, 2014), it is possible that these phenomena involve $\mathrm{PH}$ circuits.

Projections from the $\mathrm{PH}$ to the $\mathrm{PVN}$ and SON targeted neuropeptidergic cell groups, as determined by appositions onto CRH-, AVP-, and oxytocin-labeled neurons. Based on previous studies (Ulrich-Lai et al, 2011), appositions onto $\mathrm{CRH}$ neurons are presumed to be glutamatergic and would be predicted to excite the HPA axis. Given the actions of AVP, PH innervation of AVP neurons likely stimulates ACTH release as well as pressor responses, and may also be involved in aggressive behaviors (Dicker and Nunn, 1957; Ferris et al, 1997; Saffran and Schally, 1956). Oxytocin modulates a variety of behaviors, generally promoting social attachment and reducing anxiety (Insel, 2010; Knobloch et al, 2012; Lukas et al, 2011). Interactions between the $\mathrm{PH}$ and oxytocin cell groups could provide an additional mechanism for the effects observed after $\mathrm{PH}$ inhibition/ disinhibition. However, the neuroendocrine and behavioral data are more fitting with $\mathrm{PH}$ activation of $\mathrm{CRH}$ and AVP neurons.

In our studies of HPA axis stress responses, inhibition of the $\mathrm{PH}$ decreased $\mathrm{ACTH}$ responses to stress without affecting corticosterone release. This dissociation is not uncommon and is mediated by an increase in adrenal responsivity (Ulrich-Lai and Engeland, 2002). Enhanced sensitivity of the adrenal gland is proposed as a sympatheticmediated compensation for low ACTH in the face of stress. Conversely, disinhibition of the $\mathrm{PH}$ increased both ACTH and corticosterone responses to restraint; although, the potentiation of CORT preceded the effects on ACTH, providing another instance of a potential autonomic-based ACTH-glucocorticoid dissociation (Bornstein et al, 2008). Disinhibition of the $\mathrm{PH}$ in unstressed animals markedly increased cellular activation in the PVN, indicating a functional excitation of the PVN by $\mathrm{PH}$ output. In aggregate, these data provide evidence that the $\mathrm{PH}$ stimulates HPA axis stress responses, particularly the initial hypothalamicpituitary neuroendocrine outflow. The sufficiency of the $\mathrm{PH}$ to stimulate neuroendocrine stress responses at all levels of the HPA axis (PVN, ACTH, and corticosterone) suggest this is a novel site for driving stress sensitization. The effects of $\mathrm{GABA}_{\mathrm{A}}$ antagonism to facilitate stress responding also reveal the importance of tonic inhibition within the $\mathrm{PH}$. This inhibition appears to be mediated by local inhibitory $\mathrm{PH}$ neurons that dampen stress-excitatory output. Further, IL projections to the hypothalamus are predominantly glutamatergic (Myers et al, 2014) and target these PH GABAergic neurons. Alternatively, hypothalamic-projecting neurons of the LS are GABAergic, as are a portion of MeA neurons (Myers et al, 2014). Thus, in addition to local PH GABAergic neurons, integration of forebrain GABAergic inputs to the $\mathrm{PH}$ may also contribute to stress responsiveness.

In conclusion, the $\mathrm{PH}$ represents an important integrative center linking the stress-regulatory influence of the limbic forebrain with anxiety-like avoidance behavior and neuroendocrine systems. The vast interconnectedness of the $\mathrm{PH}$ with cell groups mediating behavioral and physiological processes suggests that the $\mathrm{PH}$ may be uniquely positioned to play a role in stress pathologies associated with altered limbic functional connectivity.

\section{FUNDING AND DISCLOSURE}

This work was supported by National Institutes of Health (NIH) grants MH049698 and MH069860 to J. P. Herman, as well as NIH grants DK059803, HL122454, and an American Heart Association fellowship to B. Myers. The authors declare no conflicts of interest.

\section{ACKNOWLEDGMENTS}

We are grateful for technical assistance from Kenneth Jones, C. Mark Dolgas, Benjamin Packard, Jessica M. McKlveen, and Rachel Morano.

\section{REFERENCES}

Abrahamson EE, Moore RY (2001). The posterior hypothalamic area: chemoarchitecture and afferent connections. Brain Res $\mathbf{8 8 9}$ : $1-22$.

Belzung C, Griebel G (2001). Measuring normal and pathological anxiety-like behaviour in mice: a review. Behav Brain Res 125: 141-149.

Bornstein SR, Engeland WC, Ehrhart-Bornstein M, Herman JP (2008). Dissociation of ACTH and glucocorticoids. Trends Endocrinol Metab 19: 175-180. 
Coolen LM, Wood RI (1998). Bidirectional connections of the medial amygdaloid nucleus in the Syrian hamster brain: simultaneous anterograde and retrograde tract tracing. J Comp Neurol 399: 189-209.

Davis M (1997). Neurobiology of fear responses: the role of the amygdala. J Neuropsychiatry Clin Neurosci 9: 382-402.

Dicker SE, Nunn J (1957). Fate and excretion of the pressor activity of vasopressin in rats. J Physiol 138: 11-18.

DiMicco JA, Abshire VM, Hankins KD, Sample RH, Wible JH Jr (1986). Microinjection of GABA antagonists into posterior hypothalamus elevates heart rate in anesthetized rats. Neuropharmacology 25: 1063-1066.

Ebner K, Rupniak NM, Saria A, Singewald N (2004). Substance P in the medial amygdala: emotional stress-sensitive release and modulation of anxiety-related behavior in rats. Proc Natl Acad Sci USA 101: 4280-4285.

Ferris CF, Melloni RH, Koppel G, Perry KW, Fuller RW, Delville Y (1997). Vasopressin/serotonin interactions in the anterior hypothalamus control aggressive behavior in golden hamsters. J Neurosci 17: 4331-4340.

File SE, Lippa AS, Beer B, Lippa MT (2004). Animal tests of anxiety. Curr Protoc Neurosci 8: 1-22.

Flak JN, Solomon MB, Jankord R, Krause EG, Herman JP (2012). Identification of chronic stress-activated regions reveals a potential recruited circuit in rat brain. Eur J Neurosci 36: 2547-2555.

Hamani C, Diwan M, Macedo CE, Brandão ML, Shumake J, Gonzalez-Lima F et al (2010). Antidepressant-like effects of medial prefrontal cortex deep brain stimulation in rats. Biol Psychiatry 67: 117-124.

Herman JP (2013). Neural control of chronic stress adaptation. Front Behav Neurosci 7: 61.

Herman JP, Figueiredo H, Mueller NK, Ulrich-Lai Y, Ostrander MM, Choi DC et al (2003). Central mechanisms of stress integration: hierarchical circuitry controlling hypothalamopituitary-adrenocortical responsiveness. Front Neuroendocr 24: $151-180$.

Insel TR (2010). The challenge of translation in social neuroscience: a review of oxytocin, vasopressin, and affiliative behavior. Neuron 65: 768-779.

Knobloch HS, Charlet A, Hoffmann LC, Eliava M, Khrulev S, Cetin AH et al (2012). Evoked axonal oxytocin release in the central amygdala attenuates fear response. Neuron 73: 553-566.

Krause EG, Kloet AD, de Flak JN, Smeltzer MD, Solomon MB, Evanson NK et al (2011). Hydration state controls stress responsiveness and social behavior. J Neurosci 31: 5470-5476.

Lisa M, Marmo E, Wible JH Jr., DiMicco JA (1989). Injection of muscimol into posterior hypothalamus blocks stress-induced tachycardia. Am J Physiol 257: R246-R251.

Lukas M, Toth I, Reber SO, Slattery DA, Veenema AH, Neumann ID (2011). The neuropeptide oxytocin facilitates prosocial behavior and prevents social avoidance in rats and mice. Neuropsychopharmacology 36: 2159-2168.

McKlveen JM, Myers B, Flak JN, Bundzikova J, Solomon MB, Seroogy KB et al (2013). Role of prefrontal cortex glucocorticoid receptors in stress and emotion. Biol Psychiatry 74: 672-679.

Myers B, Dolgas CM, Kasckow J, Cullinan WE, Herman JP (2014). Central stress-integrative circuits: forebrain glutamatergic and
GABAergic projections to the dorsomedial hypothalamus, medial preoptic area, and bed nucleus of the stria terminalis. Brain Struct Funct 219: 1287-1303.

Myers B, McKlveen JM, Herman JP (2012). Neural regulation of the stress response: the many faces of feedback. Cell Mol Neurobiol 32: 683-694.

Myers B, McKlveen JM, Herman JP (2014). Glucocorticoid actions on synapses, circuits, and behavior: implications for the energetics of stress. Front Neuroendocrinol 35: 180-196.

Phelps EA, LeDoux JE (2005). Contributions of the amygdala to emotion processing: from animal models to human behavior. Neuron 48: 175-187.

Saffran M, Schally AV (1956). Effect of histamine, hog vasopressin, and corticotropin-releasing factor (CRF) on ACTH release in vitro. Proc Soc Exp Biol Med 92: 636-637.

Schnurr R (1972). Localization of the septal rage syndrome in LongEvans rats. J Comp Physiol Psychol 81: 291-296.

Shekhar A, DiMicco JA (1987). Defense reaction elicited by injection of GABA antagonists and synthesis inhibitors into the posterior hypothalamus in rats. Neuropharmacology 26: 407-417.

Shekhar A, Hingtgen JN, DiMicco JA (1987). Selective enhancement of shock avoidance responding elicited by GABA blockade in the posterior hypothalamus of rats. Brain Res 420: 118-128.

Singewald GM, Rjabokon A, Singewald N, Ebner K (2011). The modulatory role of the lateral septum on neuroendocrine and behavioral stress responses. Neuropsychopharmacology 36: 793-804.

Sotres-Bayon F, Quirk GJ (2010). Prefrontal control of fear: more than just extinction. Curr Opin Neurobiol 20: 231-235.

Sudheimer K, Keller J, Gomez R, Tennakoon L, Reiss A, Garrett A et al (2015). Decreased hypothalamic functional connectivity with subgenual cortex in psychotic major depression. Neuropsychopharmacology 40: 849-860.

Swanson LW (2004). Brain Maps: Structure of the Rat Brain (3rd edition).

Thompson RH, Swanson LW (2010). Hypothesis-driven structural connectivity analysis supports network over hierarchical model of brain architecture. Proc Natl Acad Sci USA 107: 15235-15239.

Ulrich-Lai YM, Engeland WC (2002). Adrenal splanchnic innervation modulates adrenal cortical responses to dehydration stress in rats. Neuroendocrinology 76: 79-92.

Ulrich-Lai YM, Herman JP (2009). Neural regulation of endocrine and autonomic stress responses. Nat Rev Neurosci 10: 397-409.

Ulrich-Lai YM, Jones KR, Ziegler DR, Cullinan WE, Herman JP (2011). Forebrain origins of glutamatergic innervation to the rat paraventricular nucleus of the hypothalamus: differential inputs to the anterior versus posterior subregions. J Comp Neurol 519: 1301-1319.

Veenman CL, Reiner A, Honig MG (1992). Biotinylated dextran amine as an anterograde tracer for single- and double-labeling studies. J Neurosci Methods 41: 239-254.

Vertes RP (2004). Differential projections of the infralimbic and prelimbic cortex in the rat. Synapse 51: 32-58.

Ziegler DR, Cullinan WE, Herman JP (2002). Distrubuion of vesicular glutamate transporter mRNA in rat hypothalamus. J Comp Neurol 448: 217-229.

Supplementary Information accompanies the paper on the Neuropsychopharmacology website (http://www.nature.com/npp) 